\title{
Dreijahresergebnisse der deutschlandweiten Umfrage zu Augenverletzungen durch Feuerwerkskörper
}

\author{
Ameli Gabel-Pfisterer ${ }^{a}$ Daniel Böhringer ${ }^{b} \quad$ Hansjuergen Agostini ${ }^{b}$ \\ aAugenklinik, Klinikum Ernst-von-Bergmann, Potsdam, Deutschland; \\ ${ }^{b}$ Klinik für Augenheilkunde, Medizinische Fakultät, Universitätsklinikum Freiburg, Freiburg im Breisgau, Deutschland
}

\section{Schlüsselwörter}

Feuerwerk · Verletzungen · Auge · Sprengkörper · Registerstudie

\begin{abstract}
Zusammenfassung
Hintergrund: Weltweit entstehen an Feiertagen schwere Augenverletzungen durch Feuerwerkskörper. Die Datenlage für Deutschland ist bislang unklar.

Fragestellung: Wie häufig entstehen solche Verletzungen in Deutschland, wer ist gefährdet, wie schwer sind die Verletzungen, welche Therapien erfordern sie, und wie häufig treten Begleitverletzungen auf?
\end{abstract}

Methode: Deutschlandweite standardisierte Online-Umfrage an bettenführenden, notdienstleistenden Augenkliniken und Auswertung der Ergebnisse über 3 Jahre.
Ergebnisse: Die Beteiligung konnte von 2016 bis 2019 von 41 auf 51 Augenkliniken gesteigert werden. Von 1356 Patienten war mehr als ein Drittel (33-39\%) minderjährig, rund 60\% 25 Jahre oder jünger und rund 60\% Bystander. Ein Viertel der Verletzungen war schwer und erforderte eine stationäre Therapie. Begleitverletzungen am Partnerauge, an Händen und Gesicht waren bei Minderjährigen häufiger als bei Erwachsenen. Bulbusrupturen wurden bei 10 Kindern und 38 Erwachsenen dokumentiert.

Schlussfolgerungen: Vor allem Minderjährige und Zuschauer müssen besser geschützt werden.

(c) 2019 Der/die Autor(en)
Weltweit entstehen an Silvester und anderen Feiertagen vor allem durch privat gezündete Feuerwerkskörper schwere Verletzungen vor allem an den Händen, dem Gesicht und den Augen. Häufig betroffen sind Kinder und junge Männer. In vielen Fällen werden zudem unbeteiligte Zuschauer oder Passanten sogenannte «Bystander», verletzt. Die Augenverletzungen werden in rund 20\% der Fälle als schwer eingestuft und sind dann durch eine schlechte funktionelle Prognose gekennzeichnet.

Der Dachverband der nationalen augenärztlichen Gesellschaften, das International Council of Ophthalmology (ICO), hat seine Mitglieder im Mai 2016 aufgerufen, sich in der Diskussion um ein Verbot privat genutzter Feuerwerks- und Knallkörper zu engagieren, um die Zahlen dieser vermeidbaren Verletzungen zu reduzieren [10]. Um dieser Diskussion eine fundierte Datenbasis zu geben, hat die Deutsche Ophthalmologische Gesellschaft (DOG) deutsch- landweit Daten über Augenverletzungen durch Feuerwerks- und Knallkörper erhoben. Regelmäßig wiederholte Umfragen sind sinnvoll, um die Inzidenz solcher Verletzungen zu bestimmen und Einflüsse von gesetzlichen Regelungen beurteilen zu können. So ist etwa in Deutschland nach Umsetzung der europäischen Richtlinie 2007/23/EG zur Harmonisierung des Handels seit 2009 die Menge des erlaubten Schwarzpulvers in Silvesterraketen von $6 \mathrm{~g}$ je Rakete auf $10 \mathrm{~g}$ je Rakete angehoben worden, was nach Unterlauft in den Folgejahren zu einem Anstieg der Augenverletzungen geführt hat [21], während eine Freiburger Studie diesen Effekt nicht beobachtet hatte [9]. Gesellschaftliche Verhaltensveränderungen wie absichtliches Abfeuern auf Unbeteiligte können ebenfalls nur mit longitudinalen Daten erkannt und bewertet werden.

Das deutsche Sprengstoffgesetz definiert Feuerwerkskörper als «pyrotechnische Artikel für Unterhaltungszwecke» und unter- 


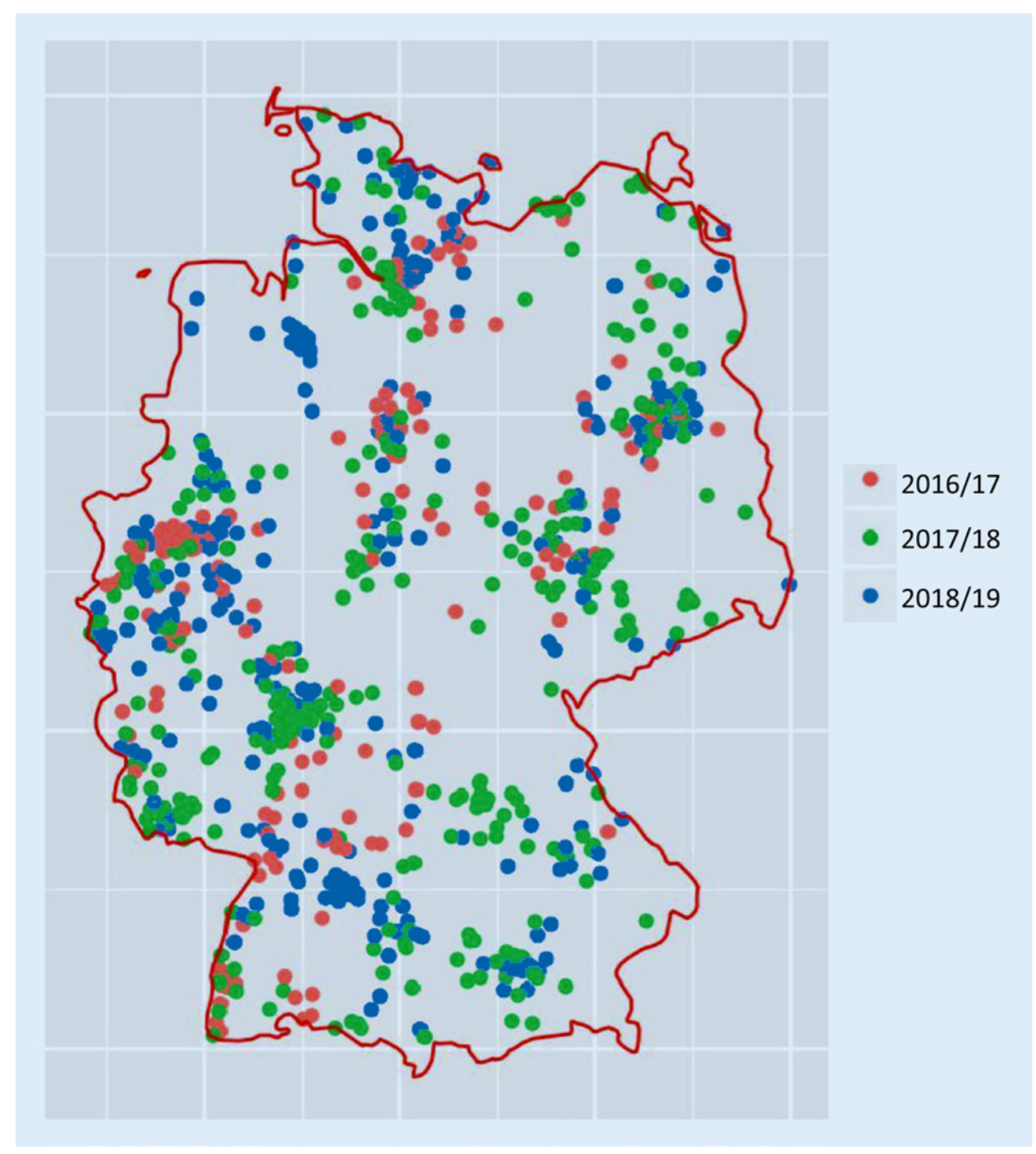

Abb. 1. Verteilung der Verletzungen über Deutschland über die 3 Jahreswechsel 2016 bis 2019 . Ballungsräume sind bevorzugt betroffen.

teilt diese nach dem Gefahrenpotenzial, dem Lärmpegel und dem vorgesehenen Verwendungsort in die Kategorien F1 bis F4. Bei dieser Einteilung werden jeweils der sachgerechte Gebrauch und insbesondere die Einhaltung des Sicherheitsabstands vorausgesetzt. Feuerwerkskörper der Kategorie F1 sind Personen ab 12 Jahren ganzjährig erlaubt. Für den privaten Gebrauch sind Kategorie-F2-Artikel ab dem 29.12. (im Falle eines Sonntags ab dem 28.12.) erwerbbar, abbrennen dürfen sie Personen über 18 Jahre am 31. Dezember und am 1. Januar. Kategorie-F3- und -F4-Artikel sind professionellen Pyrotechnikern vorbehalten [2].

Bisher war außerhalb lokaler Erhebungen wie aus Leipzig und Freiburg $[9,21]$ unklar, wie häufig Augenverletzungen durch Feuerwerkskörper in Deutschland auftreten, welche Personengruppen durch Feuerwerkskörper besonders gefährdet und wie schwerwiegend die Augen- und Begleitverletzungen sind. Ein besonderes Interesse gilt außerdem der Frage, ob die Betroffenen den auslösenden Feuerwerks- oder Knallkörper selbst gezündet haben oder ob sie als Zuschauer oder Passanten verletzt wurden. Die im Folgenden dargestellten Umfrageergebnisse umfassen jeweils 5 Tage um die Jahreswechsel 2016/17, 2017/18 und 2018/19.

\section{Material und Methoden}

Im Rahmen unserer prospektiven Studie baten wir alle bettenführenden Augenkliniken Deutschlands, die sich am augenärztlichen Notdienst beteiligen, um Teilnahme. Die Augenkliniken wurden dem Katalog der Deutschen Ophthalmologischen Gesellschaft entnommen. Das Anschreiben wurde via E-Mail an die jeweiligen ärztlichen Direktoren und leitenden Oberärzte versen- 


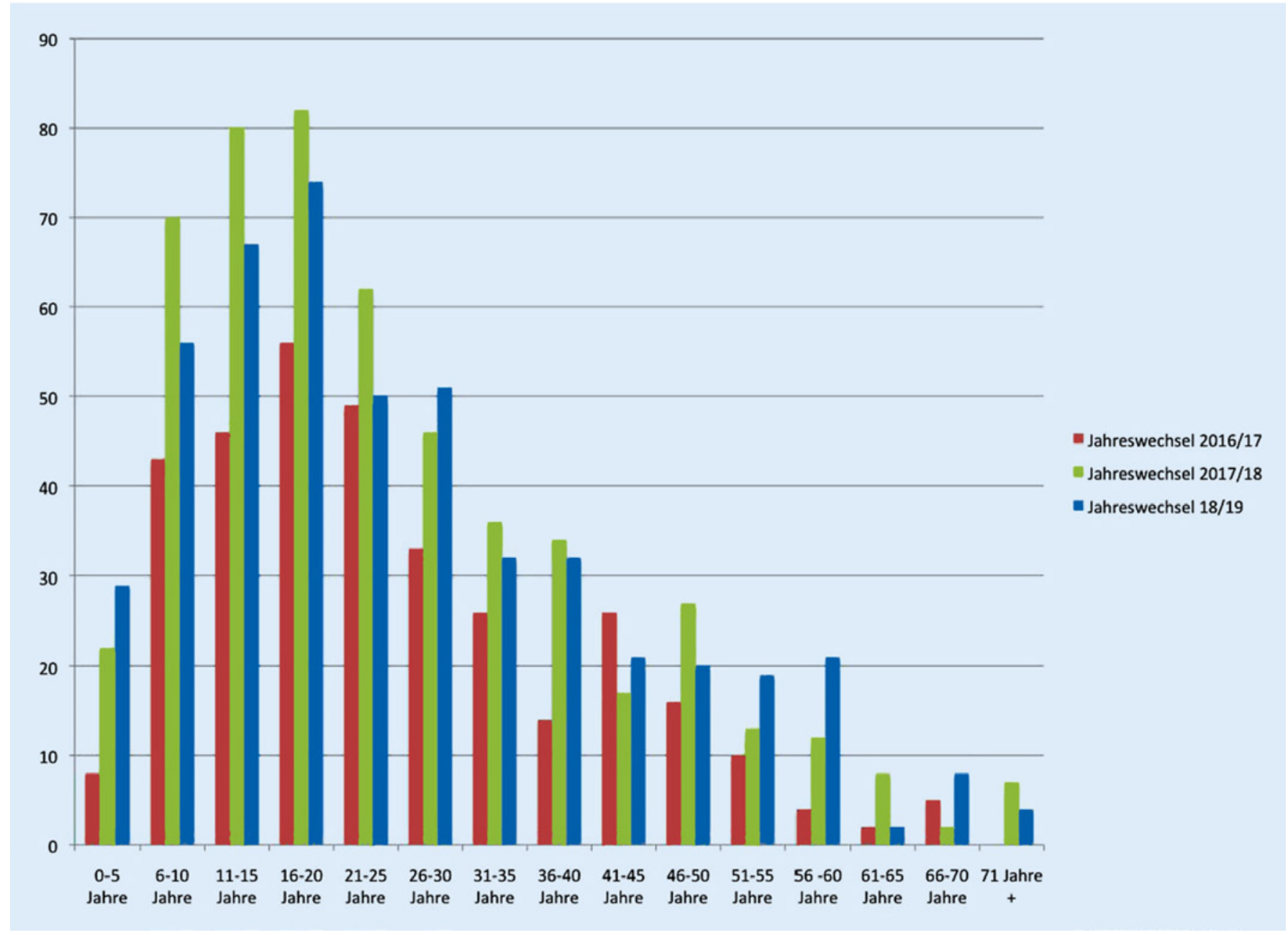

Abb. 2. Altersverteilung: absolute Zahl der Patienten je Altersgruppe. Es zeigt sich an allen 3 Jahreswechseln ein Häufigkeitsgipfel für Augenverletzungen durch Feuerwerkskörper bei Kindern, Jugendlichen und jungen Erwachsenen.

det. Bei Interessensbekundung versendeten wir personalisierte Zugangsdaten zu einem strukturierten Erfassungsbogen im Internet. Mit diesem erfassten wir Geschlecht, Lebensalter zum Zeitpunkt der Verletzung, Seitigkeit, etwaige Begleitverletzungen im Gesicht oder an den Händen, die Art der Verletzung und der erforderlichen Therapie. Außerdem erfragten wir prognostische Parameter wie abzusehender dauerhafter Visusverlust, andere Residuen wie Narbenbildungen oder die Notwendigkeit folgender ophthalmochirurgischer Folgeeingriffe. Zwei Fragen bezogen sich auf den Unfallhergang mit dem Ziel, Verursacher (selbstoder fremdgezündet einschließlich unklarer Situation) und Art des auslösenden Feuerwerkskörpers zu dokumentieren.

Die statistische Auswertung erfolgt deskriptiv. Zusätzlich wurden mittels logistischer Regression Risikofaktoren für den schwersten Verlauf mit der Notwendigkeit einer Operation unter stationären Bedingungen charakterisiert.

Ausgewertet wurde in den 2 Altersgruppen, Kinder und Jugendliche 0 bis 17 Jahre und Erwachsene 18 Jahre oder mehr, und auf der Basis der 3 aufeinanderfolgenden Jahreswechsel 2016/17, 2017/18 und 2018/19.

\section{Ergebnisse}

\section{Beteiligung}

Beginnend mit 41 teilnehmenden Zentren zum Jahreswechsel 2016/17, beteiligten sich in den Folgejahren 49 und 51 Kliniken. Dabei wurden Daten von 350 Verletzten (2016/17), 518 (2017/18) und 488 (2018/19) durch die jeweils teilnehmenden Augenkliniken in anonymisierter Form erfasst (Abb. 1).

\section{Patientenalter und Geschlechtsverteilung}

Kinder, Jugendliche und junge Erwachsene männlichen Geschlechts sind besonders häufig von Feuerwerksverletzungen betroffen. Am Jahreswechsel 2016/17 waren 33\% der Patienten Kinder und Jugendliche, am folgenden Jahreswechsel 39\% und 2018/19 37\%. Im jungen Erwachsenenalter von 18 bis 30 Jahren waren 2016/17 34\% Patienten, 2017/18 $31 \%$ und 2018/19 30\%. Die Altersverteilung findet sich in Abb. 2.

In allen 3 Jahren waren rund drei Viertel der Patienten beider Altersgruppen männlich. Um den Jahreswechsel 2016/17 waren 75\% der Minderjährigen (115 Angaben) und 72\% der Volljährigen 


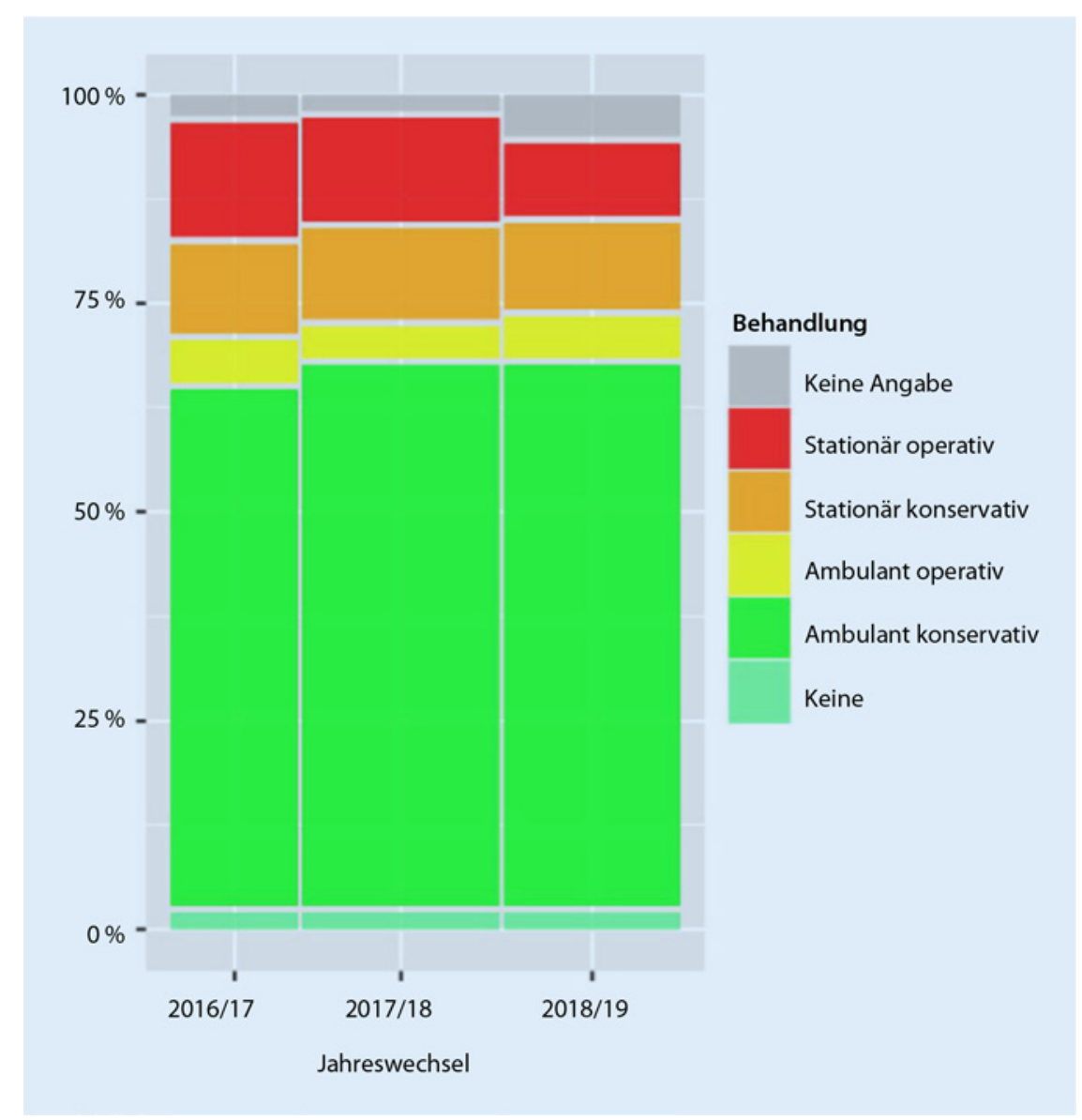

Abb. 3. Anteil der Patienten (\%) nach Therapiemodus im Jahresvergleich. An allen 3 Jahreswechseln erlitt rund ein Viertel der Patienten schwere Verletzungen, die stationär behandelt werden mussten. In 75\% der Fälle erfolgte eine ambulante Therapie. Die Breite der Balken spiegelt die Anzahl der Meldungen wider. St. $\mathrm{OP}=$ stationär operativ, St. Kons. = stationär konservativ, Amb. OP = ambulant operativ, Amb. Kons. = ambulant konservativ, Keine $=$ keine Therapie erforderlich.

männlich (220 Angaben), an Silvester 2017/18 76\% (200 Angaben) und 73\% (316 Angaben) und an Silvester 2018/19 73\% (180 Angaben) und 70\% (307 Angaben) respektive.

\section{Schweregrad der Verletzung und der Therapie}

Rund drei Viertel aller Verletzten jeden Alters wurden ambulant wegen leichter Verletzungen behandelt, während rund ein Viertel der Patienten wegen schwerer Verletzungen stationär versorgt werden musste. Zum Jahreswechsel 2016/17 benötigten 2\% ( $n$ $=7)$ aller Patienten keine Therapie, $67 \%(n=228)$ eine ambulant konservative Therapie und 6\% $(n=19)$ eine ambulant operative Therapie meist bei banalen Verletzungen der Augenoberfläche; $12 \%$ der Patienten $(n=40)$ wurden stationär konservativ bei Verletzungen der Oberfläche und stumpfen Bulbusprellungen behandelt, und $14 \%$ Patienten $(n=47)$ wurden stationär operativ meist wegen kombinierter Lid- und Oberflächenverletzungen und Bulbusverletzungen (341 Angaben) therapiert. Die Daten von Kindern, Jugendlichen und Erwachsenen und den beiden Zeiträumen (2017/18: 509 Angaben, 2018/19: 463 Angaben) unterscheiden sich dabei unwesentlich (Abb. 3).

\section{Risiko für die schweren, operativ zu versorgenden Verletzungen}

In der logistischen Regression bestätigte sich außerdem statistisch signifikant, dass Jungen und junge Männer ein höheres Risiko für die schweren, operativ zu versorgenden Verletzungen hatten. Patienten, die den auslösenden Feuerwerkskörper selbst gezündet haben, weisen statistisch ein etwas höheres Risiko für schwere, operativ zu versorgende Verletzungen auf, während keine statistische Signifikanz für das Risiko einer schweren operativ zu versorgenden Verletzung zwischen den 3 Jahren zu beobachten war.

Das logistische Regressionsmodell ist in Abb. 4 zusammengefasst. Einseitige Bulbusrupturen wurden 2016/17 bei 3 Kindern oder Jugendlichen (3\%) und 12 Erwachsenen (5\%), 2017/18 bei 6 Kindern oder Jugendlichen (3\%) und 14 Erwachsenen (4\%) und 2018/19 bei einem 8-jährigen Kind und 12 Erwachsenen dokumentiert. Über die 3 Jahre erfassten wir in Deutschland insgesamt 10 Bulbusrupturen bei Kindern und Jugendlichen. Die 2 jüngsten Kinder waren 8 Jahre alt. Das mittlere Alter lag bei 11,3 Jahren, 80\% der Patienten waren männlich, $50 \%$ hatten selbst gezündet, $50 \%$ wurden in unklarer Situation oder als Zuschauer verletzt, 50\% wurden durch Knallkörper verletzt. Für die 38 bulbuseröffnenden Schwerstverletzungen bei Erwachsenen lag das mittlere Alter bei 37,6 Jahren, $92 \%$ waren männlich, $64 \%$ hatten selbst gezündet, $40 \%$ wurden durch Raketen verletzt.

\section{Einschätzung von Visusverlust und Folgeeingriffen}

Der Erstbehandelnde schätzte das Risiko eines Visusverlust und anderer Residuen wie z.B. einer Narbenbildung ein. Bei den Patienten mit den leichten, ambulant versorgten Verletzungen betrug 


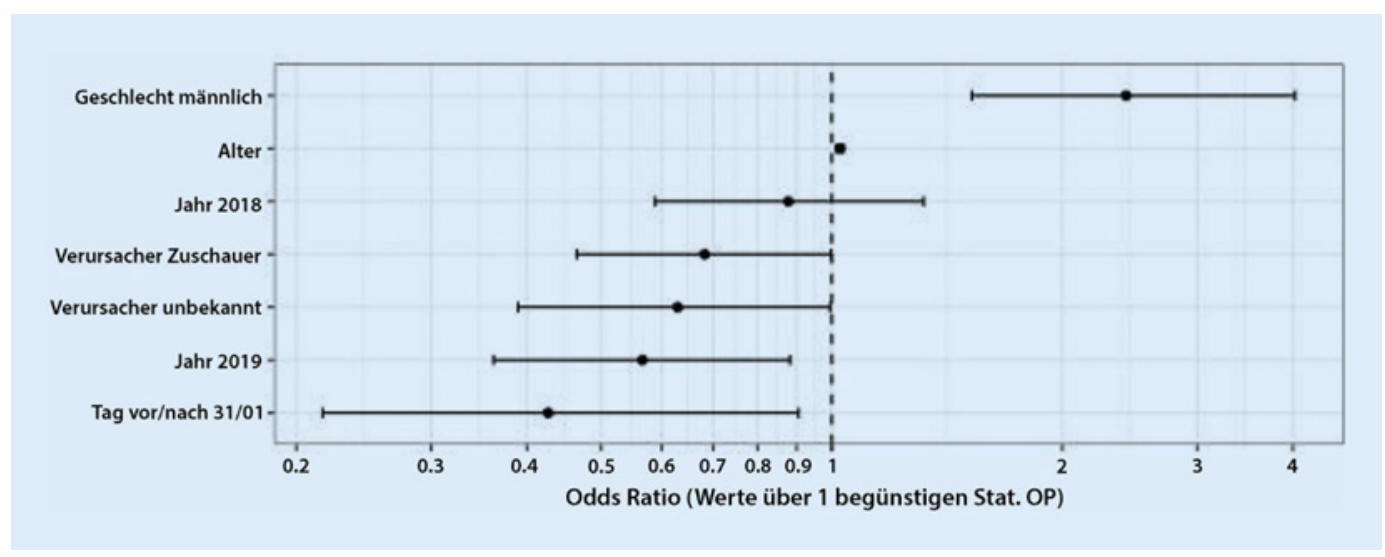

Abb. 4. Logistische Regression zur Charakterisierung von Risikofaktoren für eine schwere Augenverletzung durch Feuerwerk, die eine stationäre operative Therapie erfordert. Faktoren, deren Spannbreite die gestrichelte Linie schneidet verfehlen die statistische Signifikanz. Stat. OP = stationär operativ.

Abb. 5. Anteil der Patienten (\%) mit Folgeschäden nach Einschätzung der Prognose durch den Erstuntersucher bei leicht verletzten ambulant und schwer verletzten stationär versorgten Patienten. $\mathbf{a}$. Kinder und Jugendliche, b. Erwachsene.

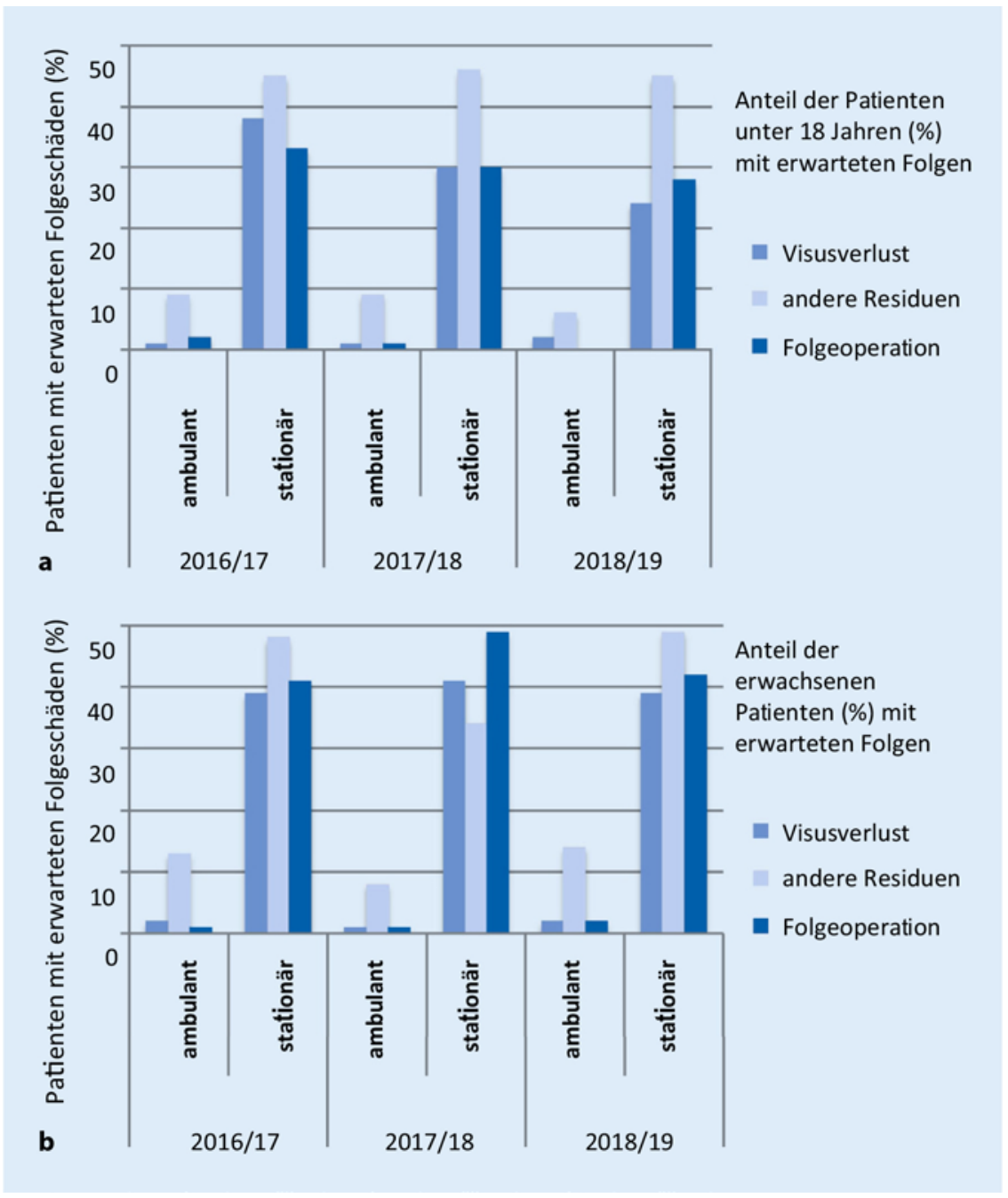

demnach das Risiko für einen Visusverlust 2016/17 1-2\% (mit anderen Residuen wie Narbenbildung an Lidern oder Hornhaut wurde bei bis zu 14\% der Patienten gerechnet).

In der Gruppe der stationär versorgten Patienten mit den schweren Verletzungen lagen diese Zahlen erwartungsgemäß deutlich höher: Mit einem dauerhaften Visusverlust wurde bei knapp 40\% der Patienten aller Altersgruppen gerechnet und mit anderen Residuen wie Narbenbildungen bei bis zu $48 \%$.

Mit Folgeeingriffen rechnete der Erstuntersucher bei 14\% der Patienten aus allen 3 Jahren, bei den Kindern und Jugendlichen mit 


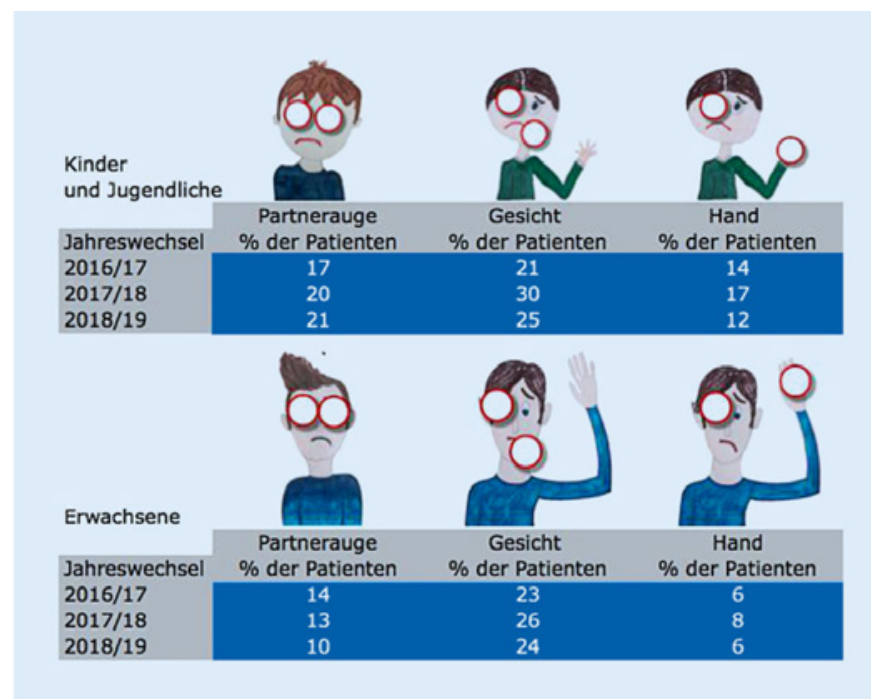

Abb. 6. Anteil der Patienten (\%) mit Begleitverletzungen am Partnerauge, im Gesicht oder an den Händen. Begleitverletzungen am Partnerauge und an den Händen waren häufiger bei Kindern und Jugendlichen.

rund 30\% etwas weniger häufig als bei den Erwachsenen, bei denen diese in über $40 \%$ der Patienten erwartet wurde (Abb. 5).

\section{Begleitverletzungen}

Beidseitige Augenverletzungen waren 2016/17 bei 17\% der Minderjährigen und bei 14\% der Erwachsenen aufgetreten, in den Folgejahren waren die Zahlen vergleichbar (Abb. 6).

Zusätzliche Gesichtsverletzungen wurden zum Jahreswechsel 2016/17 bei 21\% der verletzten Kinder und Jugendlichen und bei 23\% der verletzten Erwachsenen dokumentiert, um den Jahreswechsel 2017/18 waren 30\% der Minderjährigen und 26\% der Erwachsenen und 2018/1925 und 24\% respektive betroffen. Zusätzliche Handverletzungen waren bei den Kindern und Jugendlichen ebenfalls häufiger festzustellen. Neben der Augenverletzung erlitten zum Jahreswechsel 2016/17 14\% der Minderjährigen und 6\% der Erwachsenen Handverletzungen, im Folgejahr waren es 17\% der Minderjährigen und 8\% der volljährigen Patienten und 2018/19 12 und 6\% (Abb. 6). Besonders schwerwiegend sind Kombinationen aus beidseitigen Augenverletzungen und Gesicht- und Handverletzungen, die bei den Minderjährigen im Beobachtungszeitraum in bis zu $8 \%$ und damit 4-mal häufiger als bei den Erwachsenen (2\%) vorkamen. Andere zusätzliche Verletzungen, die nicht standardisiert dokumentiert wurden, waren z. B. offene Orbitafraktur (1 Patient), Trommelfellperforationen (2 Patienten), Thoraxverletzungen (1 Patient), Beinverletzung (1 Patientin).

\section{Unfallhergang}

\section{Zuschauer oder Passanten}

Eine Frage erfasste, ob der Verletzte den Pyrotechnikartikel selbst gezündet hatte, als Zuschauer oder Passant in Mitleidenschaft gezogen wurde oder ob die Situation in der der Unfall passierte, unklar war. Weniger als $40 \%$ der Patienten haben angegeben, den Feuerwerks- oder Knallkörper selbst gezündet zu haben (2016/17:

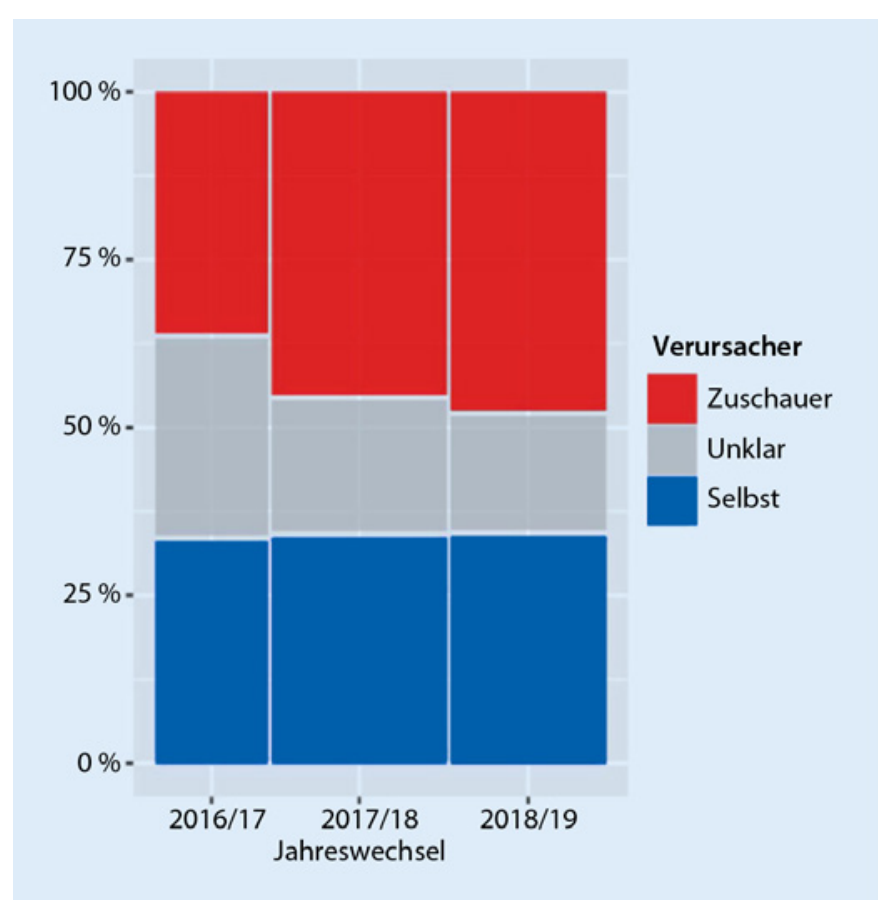

Abb. 7. Anteil der Patienten (\%) nach Unfallhergang: Über $60 \%$ der Patienten geben an, den auslösenden Feuerwerkskörper nicht selbst gezündet zu haben oder in einer unklaren Situation verletzt worden zu sein. Auch hier zeigt sich über die 3 Jahre eine hohe longitudinale Stabilität.

35\% Minderjährige, 39\% Erwachsene, 2017/18: 40\% Minderjährige und 30\% Erwachsene, 2018/19: Minderjährige 38\%, Erwachsene 33\%). Entsprechend gaben zum Jahreswechsel 2016/17 51\% der Minderjährigen und 36\% der Erwachsenen an, als Zuschauer oder Passant (2017/18: 45 und 38\% und 2018/19: 49 und 48\% respektive) oder in einer unklaren Situation (2016/17: 14 und 25\%, 2017/18: 15 und 26\% und 2018/19: 17 und 19\% respektive) verletzt worden zu sein. Dies ist in Abb. 7 zusammengefasst.

\section{Feuerwerksmittel}

Außerdem untersuchten wir, welche Art von Pyrotechnik die Verletzung verursacht hatte.

Kinder und Jugendliche wurden demnach überwiegend (in einem Drittel [2018/19] bis der Hälfte [2016/17 und 2017/18]) der Fälle von Knallkörpern verletzt, während bei den Erwachsenen Raketen (in allen 3 Jahren rund 40\%) im Vordergrund standen. Aber auch andere vermeintlich harmlose Pyrotechnik wie Bengalische Lichter oder Wunderkerzen führten in bis zu 30\% der Patienten zu Verletzungen (Abb. 8).

In einigen Fällen war jedoch nicht primär die Explosion des Knall- oder Feuerwerkskörper ursächlich: Zwei Patienten wurden durch herabfallende Raketenreste verletzt. In weiteren Fällen waren abgesprengte Teile von Pyrotechnikartikeln oder zersplitterte Brillengläser nach Aufprall Auslöser der Verletzung. Bei 2 erwachsenen Patienten und einem Kind war die Verletzung bei der Explosion eines vom Boden aufgehobenen Blindgängers am Neujahrstag entstanden. In diesen Fällen war es zu zusätzlichen Handverletzungen gekommen. 


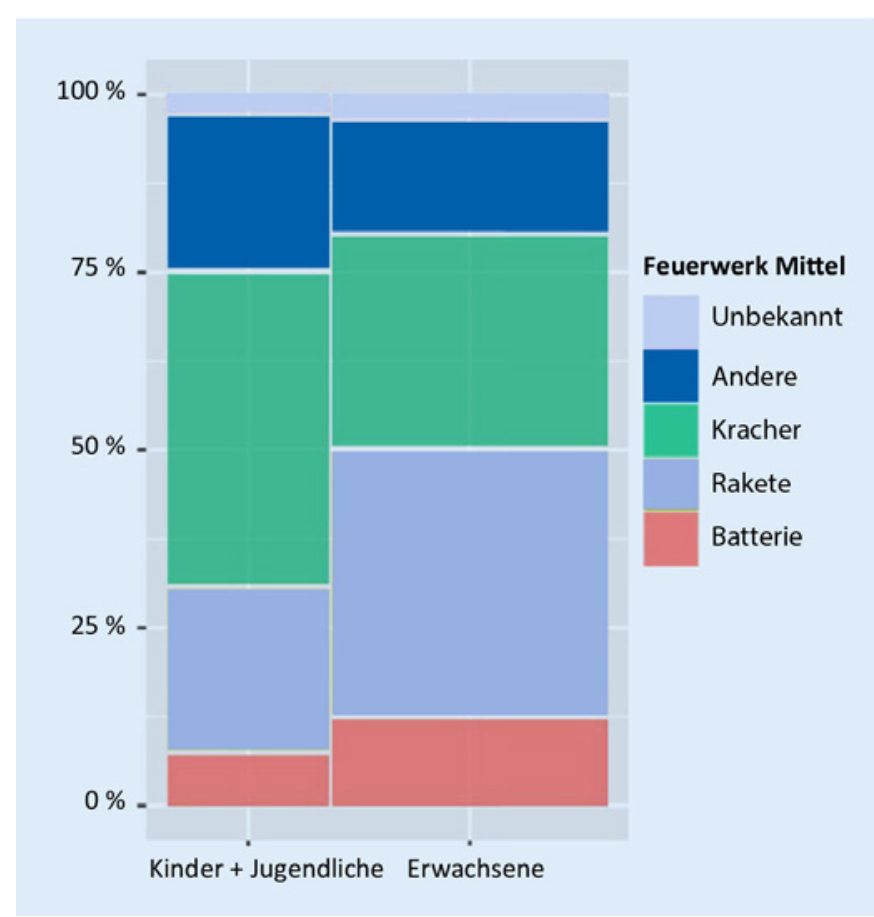

Abb. 8. Anteil der Patienten (\%) nach Art des auslösenden Feuerwerkskörpers. Kinder und Jugendliche werden häufiger als Erwachsene durch Knallkörper verletzt. Bei den Erwachsenen stehen Raketen und Batterien als Auslöser quantitativ im Vordergrund.

\section{Diskussion}

Weltweit entstehen an Silvester und anderen Feiertagen durch Feuerwerks- und Knallkörper schwere Verletzungen [3, 11, 14, 15, 19]. Besonders häufig betroffen sind dabei junge Männer [4, 7, 17, 23], die Verletzungen v. a. an den Händen, dem Gesicht und den Augen erleiden [4, 17]. Zudem werden nach einer Metaanalyse von internationalen Studien zu Augenverletzungen durch Feuerwerkskörper in rund der Hälfte der Fälle Unbeteiligte, sog. Bystander, verletzt [23]. Die Augenverletzungen werden in 18-38\% der Fälle als schwer eingestuft und sind dann durch eine schlechte funktionelle Prognose gekennzeichnet [11, 13, 21].

Bislang liegen aus Deutschland keine umfassenden Daten über Augenverletzungen, die durch Pyrotechnik hervorgerufen werden vor. In den USA hingegen werden seit dem Jahr 2000 im jährlich publizierten Feuerwerksreport aus dem National Electronic Injury Surveillance System (NEISS) der Consumer Product Safety Commission [6] Unfälle mit privater Pyrotechnik detailliert dokumentiert. In Holland und Finnland wurden in den letzten 10 Jahren ebenfalls entsprechende Register zu Augenverletzungen eingerichtet. Die daraus resultierenden Erkenntnisse haben teilweise zu einer staatlichen Einschränkung der privaten Feuerwerke geführt [24].

Ziel unserer Untersuchung war es, deutschlandweite Daten über die Patienten, Art und Schwere der Augenverletzungen und die erforderliche Therapie und den Unfallhergang zu erheben. Wir wollen damit eine verlässliche Datengrundlage schaffen, um die
Forderung des ICO nach einem Verbot von privat genutzter Pyrotechnik für Deutschland zu prüfen. Außerdem wollen wir die Dynamik dieser Verletzungen über die Jahre ermitteln, um Veränderungen der Nutzung und den Effekt von gesetzlichen Regelungen zu beurteilen.

Die Tatsache, dass viele Verletzte «Bystander» waren und viele Kinder und Jugendliche betroffen sind, lässt begründete Zweifel daran, ob die bestehenden gesetzlichen Regelungen und deren Umsetzung in Deutschland zum Schutz der Bevölkerung ausreichen.

\section{Beteiligung}

Von den 110 angefragten Kliniken erhielten wir von rund der Hälfte einen Rücklauf. Da die teilnehmenden Zentren sich gleichmäßig über das Bundesgebiet verteilen, kann davon ausgegangen werden, dass die Daten repräsentativ für Deutschland sind. Für die kommenden Umfragen bitten wir schon jetzt um rege Beteiligung.

\section{Alters- und Geschlechtsverteilung und Schwere der Verletzungen}

Die Altersverteilung der hier dokumentierten Verletzten zeigt wie nationale und internationale Studien zum Thema, dass überwiegend Kinder und Jugendliche und junge Erwachsene durch Feuerwerks- und Knallkörper verletzt werden [4, 8, 22, 23].

In unserer Studie sind im Beobachtungszeitraum rund 40\% der Verletzten Kinder und Jugendliche. Ihr Anteil an der Normalbevölkerung beträgt indes nur 16,4\% [18]. Damit ist diese Altersgruppe bei den Feuerwerksverletzungen deutlich überrepräsentiert, obwohl sie laut Sprengstoffgesetz keinen Zugang zu Pyrotechnikartikeln der Kategorie 2 haben sollte [2].

Rund $60 \%$ der Verletzten sind 30 Jahre oder jünger und stehen am Beginn ihres beruflichen Lebens. Im Fall von schweren Verletzungen, die nach unseren Daten jeden vierten Patienten betrafen, ist in rund $40 \%$ mit Einschränkungen durch dauerhafte Visusminderung oder andere Residuen zu rechnen. Unterlauft zeigte in einer detaillierten Untersuchung von Feuerwerksverletzungen aus 7 Jahren, die an der Universitätsklinik Leipzig behandelt wurden, dass 46 Augen von 50 Patienten im Falle einer operativ zu versorgenden Verletzung einen mittleren Endvisus von 0,8 $\pm 0,7$ logMar erreichten, was einem Dezimalvisus von $0,16+0,2$ entspricht; $22 \%$ der schwer verletzten Augen hatten am Ende der Nachbeobachtungszeit keine Lichtscheinwahrnehmung mehr [21]. Ausgehend von den von Unterlauft beschriebenen Verletzungsmustern, sind Vernarbungen der Lider oder der Hornhaut, Irisdefekte, aber auch Netzhautkomplikationen, Sekundärglaukom oder Bulbushypotonie möglich. Eine ähnlich ungünstige Prognose der schweren Augenverletzungen beschreiben Chang et al. in der Nachbeobachtung von 100 Patienten eines US-amerikanischen Traumazentrums, die nach Pyrotechnikverletzung der Augen im Mittel über 188 Tage nachbeobachtet wurden: Nach einer Bulbusruptur (17 Patienten) erlitten trotz meist mehrfacher Operationen 58 \% der Augen eine Erblindung auf null Lux [4]. Die Arbeitsgruppe aus Rotterdam [8] beschreibt nach Pyrotechnikverletzungen bei $38,5 \%$ der Patienten eine Visusminderung auf
176

Kompass Ophthalmol 2021;7:170-180 DOI: $10.1159 / 000520208$ 


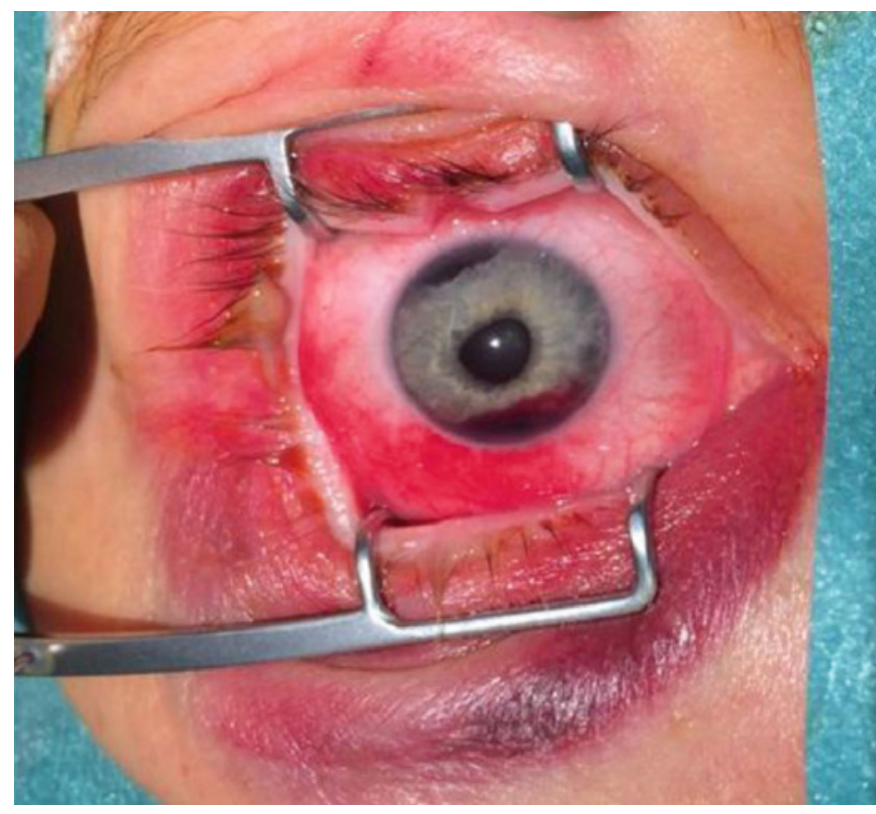

Abb. 9. Kombinierte Lid- und Bindehautverletzung mit Bulbusprellung, Irisbasisausriss und Vorderkammerblutung durch herabfallende Reste eines abgebrannten Feuerwerkskörpers.

unter 0,8 und eine Erblindung auf einen Dezimalvisus von 0,1 oder weniger in $12 \%$ der Fälle.

Der etwas höhere Anteil der stationär zu behandelnden Patienten mit schweren Verletzungen bei Unterlauft et al. (38\%) [18] und Chang et al. (28\% operative Therapie) [4], die aus der Maximalversorgung kommen, gegenüber den hier beschriebenen deutschlandweiten Zahlen (25\%), die in der Fläche erhoben wurden, ist vermutlich ein Selektionseffekt.

Über die 3 Jahre erfassten wir in Deutschland insgesamt 10 Bulbusrupturen bei Kindern und Jugendlichen und 38 dieser schwersten, prognostisch ungünstigen Verletzungen bei Erwachsenen.

Obwohl drei Viertel der Patienten ambulant zu versorgen waren, schätzten die Erstuntersucher die Visusprognose in 2\% dieser Fälle als «eingeschränkt» ein. Mögliche Residuen wurden bei jedem zehnten Patienten erwartet. Diese Einschätzungen können ein Hinweis darauf sein, dass auch banal bis mittelschwer eingeordnete Verletzungen funktionell relevant sind und dem Patienten mittelund langfristig Beschwerden bereiten können. Eine Nachbeobachtung der Patienten ist aufgrund der anonymisierten Datenerhebung dieses Registers nicht möglich, sodass die Prognose bei Erstvorstellung nicht überprüft werden kann. Dennoch sehen wir eine Vergleichbarkeit mit den oben genannten Studien, die das funktionelle Follow-up dieser Verletzungen beschreiben [4, 8, 17, 22].

Problematisch bei Verletzungen durch Feuerwerkskörper ist die häufig auftretende äußerst variable Kombination von thermischen, chemischen und mechanischen Defekten des Bulbus und der okulären Adnexe, was nicht nur die Therapie, sondern auch die standardisierte Dokumentation erschwert $([4,8,21]$, Wolf et al. in dieser Ausgabe). Dies konnte hier bestätigt werden: $85 \%$ der Patienten mit Bulbusruptur und 80\% der Patienten mit schweren Contusiones stellten sich mit einer Kombination von zusätzlichen
Lid-, Bindehaut und Hornhautverletzungen vor, die einen oft unabsehbaren Langzeitverlauf mit Narbenbildungen zeigen und spätere Reoperationen erfordern (Abb. 9).

\section{Begleitverletzungen}

Ebenso erfassten wir über alle 3 Jahre, dass fast jedes fünfte Kind eine beidseitige Augenverletzung davonträgt oder Begleitverletzungen an den Händen, in je einem Fall auch mit Finger- oder Handamputation, erleidet. Begleitverletzungen im Gesicht wurden bei einem Viertel der Minderjährigen dokumentiert und sind somit etwas häufiger als bei Erwachsenen. Die Zahlen sind durchweg höher als bei den Volljährigen, was zeigt, wie inadäquat Kinder und Jugendliche das Gefahrenpotenzial der Feuerwerkskörper einschätzen. Eine Besonderheit dieser Registerstudie ist es, dass diese Begleitverletzungen miterfasst werden. Entsprechend deutlich wird, dass mögliche Schutzmaßnahmen insbesondere für Kinder und Jugendliche umfassend sein müssen.

\section{Kinder vor allem durch Knallkörper verletzt}

Da Kinder überwiegend durch Knallkörper (Kracher) verletzt werden, wäre eine konsequentere Kontrolle des Abgabeverbots von Knallkörpern der Kategorie F2 an Minderjährige ein sinnvoller erster Schritt. Kivelä konnte zeigen, dass in Finnland Augenverletzungen und Begleitverletzungen an den Händen nach einem Verkaufsverbot von einzelnen Feuerwerksartikeln zurückgegangen sind [24].

In mehr als der Hälfte der Fälle werden Zuschauer und Passanten verletzt

Besonders dramatisch sind Augenverletzungen, die Zuschauer oder Passanten treffen. Wisse et al. zeigten in ihrer Metaanalyse einen Anteil von Verletzten Zuschauern («bystander») von 47\% (min. 14\% bis max. 80\%) [23], andere Autoren beschreiben mit rund $70 \%[8,21]$ bemerkenswert hohe Zahlen. Auch in der vorliegenden Erfassung haben über alle 3 Jahre über 60\% der Patienten angegeben, als Zuschauer oder Passant oder in einer unklaren Situation verletzt worden zu sein. Diese Zahl ist bei den Minderjährigen etwas höher als bei den Erwachsenen.

Zudem bereiten in den letzten Jahren absichtliche «Angriffe» mit Pyrotechnik auf Unbeteiligte, wie sie von 5 Patienten im Rahmen der Erhebung berichtet wurden, oder gar auf Rettungspersonal [16] zunehmend Sorgen.

\section{Was kann getan werden, um Verletzungen zu verhindern?}

Das Tragen einer Schutzbrille beim Abbrennen von Feuerwerk kann beitragen, das Risiko funktionell und ästhetisch einschränkender Verletzungen an den Augen zu verringern [20]. Die gleichzeitige Abgabe von Schutzbrillen mit Feuerwerkskörpern wird daher befürwortet ([1], Kivelä diese Ausgabe). Aufklärungskampagnen zu den Risiken von Feuerwerkskörpern in einzelnen Ländern oder Städten haben ebenfalls graduelle Verbesserungen erbracht [20], während signifikante Ergebnisse bei der Reduktion der Augenverletzungen durch Feuerwerke nur durch eine gesetzliche Beschränkung der Verfügbarkeit von Feuerwerkskörpern erzielt werden konnten [1, 3, 20, 23]. 
In den USA werden in verschiedenen Bundesstaaten verschiedene gesetzliche Regeln angewandt; auch das Spektrum der Pyrotechnik unterscheidet sich von dem in Europa üblichen. So sind dort Verletzungen durch Granaten und Granatwerfer mit schwersten Kopfverletzungen und Todesfällen beschrieben worden $[4,17]$. Die Zahlen der Verletzungen in den unterschiedlichen Bundesstaaten der USA zeigen klar, dass eine restriktive Regulation der Verfügbarkeit von Pyrotechnik zu einer deutlich niedrigeren Inzidenz von Verletzungen durch Feuerwerk führt. Die Metaanalyse von Wisse bestätigt, dass die Inzidenz durch den eingeschränkten privaten Zugang zu Feuerwerkstechnik um 87\% reduziert werden kann [23].

Die Europäische Union, die zwar aus Gründen der Marktharmonisierung die Anhebung der Sprengstoffmenge ermöglicht hat, bietet dafür die gesetzliche Grundlage, indem sie feststellt: «Die Verwendung von pyrotechnischen Gegenständen und insbesondere von Feuerwerkskörpern unterliegt in den einzelnen Mitgliedstaaten sehr unterschiedlichen kulturellen Gepflogenheiten und Traditionen. Daher ist es erforderlich, den Mitgliedstaaten unter anderem aus Gründen der öffentlichen Sicherheit oder der menschlichen Gesundheit und Sicherheit die Einführung nationaler Maßnahmen zur Beschränkung der Verwendung oder des Verkaufs bestimmter Kategorien von pyrotechnischen Gegenständen an die breite Öffentlichkeit zu ermöglichen.» [5]

Der Genuss von Alkohol [1] und anderen Drogen, wie ihn ein Patient unserer Studie, bestätigte, schränkt die situative Risikobewertung ein. Von der Einhaltung des sachgerechten Gebrauchs, z. B. des Sicherheitsabstands, wie ihn das deutsche Sprengstoffgesetz voraussetzt, kann in solchen Fällen nicht ausgegangen werden, was ebenfalls gegen die freie Abgabe von Feuerwerkskörpern der Kategorie 2 an über 18-Jährige spricht. Wir haben den Rauschmittelgenuss nicht standardmäßig erfasst und verweisen dazu auf die Erhebung von Huth et al. in dieser Ausgabe.

\section{Limitationen und Konsequenzen der Studie}

Unsere prospektive Studie zielt auf die quantitative Erfassung der Verletzungen. Damit dies zeitnah und effizient durch die in den Kliniken notdienstleistenden Augenärzte erfolgen kann, haben wir den Online-Fragebogen auf wesentliche Punkte und auf eine grobe Kategorisierung der Art der Augenverletzung, angelehnt an die Birmingham Eye trauma terminology [12], oder der Pyrotechnikartikel beschränken müssen. Wir erfassen Daten ohne Followup, sodass wir bei der Abschätzung der Folgen (Visusreduktion, Residuen oder Folgeeingriffe) auf die Erfahrung der erstuntersuchenden Kollegen angewiesen sind.

Unsere Studie beruht zudem auf freiwilliger Teilnahme und wendet sich nur an die bettenführenden Augenabteilungen und Kliniken Deutschlands und stellt daher kein komplettes Bild dar. Der Rücklauf beträgt bislang etwa 50\% der deutschen Augenkliniken, welche davon Notdienst leisten, muss in Zukunft mit erfasst werden. Die niederländische Augenärztliche Gesellschaft (NOG) kann eine deutlich höhere Beteiligung von rund 95\% an ihrem Register verzeichnen [24], ein Ziel das wir mit dieser Umfrage in den nächsten Jahren ebenfalls anstreben.
Durch die Wiederholung der Studie über 3 Jahre und die hohe Patientenzahl konnte jedoch eine hohe longitudinale Datenstabilität erzielt werden, die einen gleichbleibend hohen Anteil von betroffenen Kindern und Jugendlichen und von verletzten Zuschauern und Passanten zeigt. Diese Zahlen müssen nach Auffassung von DOG und ICO mit geeigneten gesetzgeberischen Maßnahmen reduziert werden. Durch Aufklärungskampagnen über den Umgang mit Feuerwerksartikeln und geeignete persönliche Schutzmaßnahmen kann die Sicherheit zusätzlich verbessert werden.

Augenverletzungen stellen je nach Studie 18-30\% aller Verletzungen durch Feuerwerkskörper [4, 6] dar. Aus diesen Zahlen kann man die Zahl aller Verletzungen durch Feuerwerkskörper in Deutschland nur grob abschätzen. Wir würden daher eine vergleichbare Erhebung auch für andere Fachbereiche wie Handchirurgie, Unfallchirurgie, Hals-Nasen-Ohren-Heilkunde und Mund-Kiefer-Gesichts-Chirurgie begrüßen.

Der hier verwendete Fragebogen ermöglicht die Integration der anonymisierten deutschen Daten in die Umfrage der Société ophthalmologique Européenne (SOE), die wiederum mit den Daten des ICO kompatibel ist, sodass wir die Initiative des ICO direkt unterstützen können. Wir ermöglichen so den Studiengruppenmitgliedern eine Teilnahme in allen genannten Erfassungen mit einmaliger Dateneingabe in unseren anonymisierten Online-Bogen.

\section{Resümee}

Die augenärztlichen Fachgesellschaften, allen voran die Deutsche Ophthalmologische Gesellschaft, leisten ihren Beitrag durch die Verbesserung der Datenlagen auf nationaler Ebene. Das Engagement aller Augenärzte, die sich in den vergangenen 3 Jahren an der Erfassung von Feuerwerksverletzungen in Deutschland in über 50 Zentren beteiligt haben, spricht dafür, dass das Problem als dringlich, weil vermeidbar eingeschätzt wird. Die vorliegenden Daten sprechen dafür, dass privat genutzte Feuerwerksmittel ein Gefahrenpotenzial besonders für Kinder, Jugendliche und Zuschauer oder Passanten bergen. International hat das ICO sich bereits für ein Verbot privat genutzter Feuerwerke ausgesprochen. Eine gesellschaftliche Diskussion über Nutzen und Risiko von für den privaten Gebrauch hergestellten und abgebrannten Feuerwerkskörpern, die Abwägung persönlicher Freiheit einerseits und der Gefahr für Mitmenschen und Umwelt andererseits sollte auch in Deutschland ernsthaft geführt werden.

\section{Disclosure Statement}

A. Gabel-Pfisterer, D. Böhringer und H. Agostini geben an, dass kein Interessenkonflikt besteht.

Für diesen Beitrag wurden von den Autoren keine Studien an Menschen oder Tieren durchgeführt. Für die aufgeführten Studien gelten die jeweils dort angegebenen ethischen Richtlinien.

\section{Additional information}

Aus Gründen der besseren Lesbarkeit wird in diesem Beitrag überwiegend das generische Maskulinum verwendet. Dies impliziert immer beide Formen, schließt also die weibliche Form mit ein. 


\section{Mitglieder der Feuerwerks-Verletzungen-Studiengruppe}

M. Fuest (Augenklinik, Klinikum der Medizinischen Fakultät der RWTH Aachen 17/18, 18/19); P. Walter (Augenklinik, Klinikum der Medizinischen Fakultät der RWTH Aachen 17/18, 18/19); Y. Botros (Klinikum Augsburg, Klinik für Augenheilkunde, Augsburg 16/17, 17/18, 18/19); A. Mueller (Klinikum Augsburg, Klinik für Augenheilkunde, Augsburg 16/17, 17/18, 18/19) N. Al-Ashi (Oberlausitz-Kliniken gGmbH Krankenhaus Bautzen, Augenklinik, Bautzen 16/17); M. Lenglinger (Augenklinik Universitätsklinikum Charité, Berlin 16/17, 17/18, 18/19); B. Müller (Augenklinik Universitätsklinikum Charité, Berlin 16/17, 17/18, 18/19); S. Schönfeld (Augenklinik Universitätsklinikum Charité, Berlin 16/17, 17/18, 18/19); I. Seibel (Augenklinik Univer sitätsklinikum Charité, Berlin 16/17, 17/18, 18/19); A. Joussen (Augenklinik Universitätsklinikum Charité, Berlin 16/17, 17/18, 18/19); H. Breuß (Augen klinik, Helios Klinikum Berlin-Buch, Berlin 16/17, 17/18, 18/19); J. Kuchenbecker (Augenklinik, Helios Klinikum Berlin-Buch, Berlin 16/17, 17/18 18/19); M.H. Foerster (Augenklinik, Helios Klinikum Berlin-Buch, Berlin 16/17, 17/18, 18/19); A. Berthold (Augenklinik Berlin-Marzahn GmbH, Berlin 16/17, 17/18, 18/19); C. Wirbelauer (Augenklinik Berlin-Marzahn GmbH Berlin 16/17, 17/18, 18/19); H. Hofmayer (St. Gertrauden Krankenhaus, Klinik für Augenheilkunde, Berlin 16/17, 17/18, 18/1); J. Wachtlin (St. Gertrauden Krankenhaus, Klinik für Augenheilkunde, Berlin 16/17, 17/18, 18/1); K Palka (Vivantes Klinikum Neukölln, Klinik für Augenheilkunde, Berlin 16/17, 17/18, 18/19); M. Niemeyer (Vivantes Klinikum Neukölln, Klinik für Augenheilkunde, Berlin 16/17, 17/18, 18/19); T. Walla (Vivantes Klinikum Neukölln, Klinik für Augenheilkunde, Berlin 16/17, 17/18, 18/19); D. Pham (Vivantes Klinikum Neukölln, Klinik für Augenheilkunde, Berlin 16/17, 17/18, 18/19); S. Aisenbrey (Vivantes Klinikum Neukölln, Klinik für Augenheilkunde, Berlin 16/17, 17/18, 18/19); A. Tatsiou (Klinikum Bielefeld Rosenhöhe, Augenheilkunde, Bielefeld 16/17); A. Walch (Klinikum Bielefeld Rosenhöhe, Augenheilkunde, Bielefeld 16/17); R. Burk (Klinikum Bielefeld Rosenhöhe, Augenheilkunde, Bielefeld 16/17); T. Schultz (Universitäts-Augenklinik Bochum, Bochum 16/17, 17/18); N. Tsiampalis (Universitäts-Augenklinik Bochum, Bochum 16/17, 17/18); J. Rehmann (Universitäts-Augenklinik Bochum, Bochum 16/17, 17/18); U. Sliwowska (Universitäts-Augenklinik Bochum, Bochum 16/17, 17/18); M. Schojai (Universitäts-Augenklinik Bochum, Bochum 16/17, 17/18); K. Schulze (Universitäts-Augenklinik Bochum, Bochum 16/17, 17/18); N. Kamguia (UniversitätsAugenklinik Bochum, Bochum 16/17, 17/18), C. Wirtz (Universitäts-Augenklinik Bochum, Bochum 16/17, 17/18); B. Dick (Universitäts-Augenklinik Bochum, Bochum 16/17, 17/18); D. Voelker (Universitäts-Augenklinik Bonn, Bonn 16/17, 17/18, 18/19); M. Wintergerst (Universitäts-Augenklinik Bonn, Bonn 16/17, 17/18, 18/19); M. Pfau (Universitäts-Augenklinik Bonn, Bonn 16/17, 17/18, 18/19); C. Melzer (Universitäts-Augenklinik Bonn, Bonn 16/17, 17/18, 18/19); D. Hoegen (Universitäts-Augenklinik Bonn, Bonn 16/17, 17/18 18/19); F. Bosch (Universitäts-Augenklinik Bonn, Bonn 16/17, 17/18, 18/19), J.C. Andresen (Universitäts-Augenklinik Bonn, Bonn 16/17, 17/18, 18/19); T Krohne (Universitäts-Augenklinik Bonn, Bonn 16/17, 17/18, 18/19); F. Holz (Universitäts-Augenklinik Bonn, Bonn 16/17, 17/18, 18/19); M. Kathke (Städtisches Klinikum Brandenburg GmbH, Klinik für Augenheilkunde, Brandenburg 16/17, 17/18, 18/19); A. Sturm (Städtisches Klinikum Brandenburg GmbH, Klinik für Augenheilkunde, Brandenburg 16/17, 17/18, 18/19); W. Noske (Städtisches Klinikum Brandenburg GmbH, Klinik für Augenheilkunde, Brandenburg 16/17, 17/18, 18/19); T. Hübner (Augenklinik, Klinikum Bremen-Mitte 17/18); S. Brandner (Augenklinik, Klinikum Bremen-Mitte 17/18); M. Feldmann (Augenklinik, Klinikum Bremen-Mitte 17/18); J. Morsek (Augenklinik, Klinikum Bremen-Mitte 17/18); O. Rainer (Augenklinik Klinikum Bremen-Mitte 17/18); H. Bartsch (Augenklinik, Klinikum Bremen-Mitte 17/18); K. Ewald (Augenklinik, Klinikum Bremen-Mitte 17/18) E. Chankiewitz (Augenklinik, Klinikum Bremen-Mitte 17/18); T. Siegmund (St. Joseph-Stift, Augenklinik, Bremen 16/17, 17/18, 18/19); A. Bohlen (St. Joseph-Stift, Augenklinik, Bremen 16/17, 17/18, 18/19); A. Mohr (St. JosephStift, Augenklinik, Bremen 16/17, 17/18, 18/19); J. Hecker (Klinikum Chemnitz gGmbH, Klinik für Augenheilkunde, Chemnitz 16/17, 17/18, 18/19); P. Strassburger (Klinikum Chemnitz gGmbH, Klinik für Augenheilkunde, Chemnitz 16/17, 17/18, 18/19); O. Furashova (Klinikum Chemnitz gGmbH Klinik für Augenheilkunde, Chemnitz 16/17, 17/18, 18/19); K. Engelmann (Klinikum Chemnitz gGmbH, Klinik für Augenheilkunde, Chemnitz 16/17 17/18, 18/19); A. Krieb (Klinikum Darmstadt, Klinik für Augenheilkunde, Darmstadt 16/17, 18/19); K. Emmerich (Klinikum Darmstadt, Klinik für Augenheilkunde, Darmstadt 16/17, 18/19); L. Grajewski (Städtisches Klinikum Dessau, Klinik für Augenheilkunde, Dessau 16/17, 17/18, 18/19); L. Krause
(Städtisches Klinikum Dessau, Klinik für Augenheilkunde, Dessau 16/17, 17/18, 18/19); A. Jabur (Klinikum Dortmund gGmbH, Klinikzentrum Mitte, Augenheilkunde, Dortmund 16/17); K. Rüdiger (Klinikum Dortmund gGmbH, Klinikzentrum Mitte, Augenheilkunde, Dortmund 16/17); F. Lehmann (Augenklinik Klinikum Friedrichstadt Dresden 16/17, 17/18, 18/19); H. Sachs (Augenklinik Klinikum Friedrichstadt Dresden 16/17, 17/18, 18/19); E. Matthee (Augenklinik der Universität Dresden 17/18); F. Steindorf (Universitäts-Augenklinik Düsseldorf, Düsseldorf 16/17, 17/18, 18/19); C. Schnitzler (Universitäts-Augenklinik Düsseldorf, Düsseldorf 16/17, 17/18, 18/19); I. Neumann (Universitäts-Augenklinik Düsseldorf, Düsseldorf 16/17 17/18, 18/19); J. Korbmacher (Universitäts-Augenklinik Düsseldorf, Düsseldorf 16/17, 17/18, 18/19); G. Geerling (Universitäts-Augenklinik Düsseldorf, Düsseldorf 16/17, 17/18, 18/19); J. Doulgkeridis (Augenklinik, Evangelisches Krankenhaus Duisburg 17/18, 18/19); J. Erhard (Augenklinik, Evangelisches Krankenhaus Duisburg 17/18, 18/19); K. Dahms-Dowidat (Augenklinik Klinikum Barnim, Eberswalde 17/18, 18/19); T. Kotiasvili (Augenklinik Klinikum Barnim, Eberswalde 17/18, 18/19); B. v Jagow (Augenklinik Klinikum Barnim, Eberswalde 17/18, 18/19); A. Lieder (Helios Klinikum Erfurt, Klinik für Augenheilkunde, Erfurt 16/17, 17/18); M. Blum (Helios Klinikum Erfurt, Klinik für Augenheilkunde, Erfurt 16/17, 17/18); H. Westerkemper (Universitätsklinikum Essen, Essen 16/17, 17/18, 18/19); M. Böhm (Universitätsklinikum Essen, Essen 16/17, 17/18, 18/19); N. Bornfeld (Universitätsklinikum Essen, Essen 16/17, 17/18, 18/19); N. Bechrakis (Universitätsklinikum Essen, Essen 16/17, 17/18, 18/19); M. Schultheiss (Augenklinik, Evangelisches Krankenhaus Essen-Weerden 17/18, 18/19); A. Scheider (Augenklinik, Evangelisches Krankenhaus Essen-Weerden 17/18, 18/19); M. Müller (Johann Wolfgang Goethe-Universität Frankfurt, Klinik für Augenheilkunde, Frankfurt am Main 16/17, 17/18, 18/19); T. Kohnen (Johann Wolfgang Goethe-Universität Frankfurt, Klinik für Augenheilkunde, Frankfurt am Main 16/17, 17/18, 18/19); T. Eckert (Augenklinik, Klinikum Frankfurt-Höchst 17/18); C. Eckardt (Augenklinik, Klinikum Frankfurt-Höchst 17/18); M. Wisniewska (Klinikum Frankfurt Oder GmbH, Klinik für Augenheilkunde, Frankfurt Oder 16/17, 17/18); A. Just (Klinikum Frankfurt Oder GmbH, Klinik für Augenheilkunde, Frankfurt Oder 16/17, 17/18); V. Jehle (Augenklinik Universitätsklinikum Freiburg, Freiburg i. Breisgau, 16/17, 17/18, 18/19); T. Reinhard (Augenklinik Universitätsklinikum Freiburg, Freiburg i. Breisgau, 16/17, 17/18, 18/19); J. Seewald (Augenklinik, SRH Waldklinikum Gera 17/18, 18/19); C. Mais (Klinik u. Poliklinik für Augenheilkunde, Universität Gießen, Gießen 16/17, 17/18, 18/19); S. Basiakos (Klinik u. Poliklinik für Augenheilkunde, Universität Gießen, Gießen 16/17, 17/18, 18/19); B. Osman (Klinik u. Poliklinik für Augenheilkunde, Universität Gießen, Gießen 16/17, 17/18, 18/19); E. Xanthopoulou (Klinik u. Poliklinik für Augenheilkunde, Universität Gießen, Gießen 16/17, 17/18, 18/19); B. Friedburg (Klinik u. Poliklinik für Augenheilkunde, Universität Gießen, Gießen 16/17, 17/18, 18/19); M. Graef (Klinik u. Poliklinik für Augenheilkunde, Universität Gießen, Gießen 16/17, 17/18, 18/19); B. Lorenz (Klinik u. Poliklinik für Augenheilkunde, Universität Gießen, Gießen 16/17, 17/18, 18/19); U. Just (Augenklinik, Klinikum Glauchau 17/18); S. Naxer (Universitätsmedizin Göttingen-Augenklinik, Göttingen 16/7, 17/18, 18/19); C. Oterendorp (Universitätsmedizin Göttingen-Augenklinik, Göttingen 16/7, 17/18, 18/19); S. Bemme (Universitätsmedizin Göttingen-Augenklinik, Göttingen 16/7, 17/18, 18/19); J. Callizo (Universitätsmedizin Göttingen-Augenklinik, Göttingen 16/7, 17/18, 18/19); N. Feltgen (Universitätsmedizin Göttingen-Augenklinik, Göttingen 16/7, 17/18, 18/19); H. Hoerauf (Universitätsmedizin Göttingen-Augenklinik, Göttingen 16/7, 17/18, 18/19); A. Menges (Klinik und Poliklinik für Augenheilkunde, Universitätsklinikum Greifswald, Greifswald 16/17, 17/18, 18/19); F. Tost (Klinik und Poliklinik für Augenheilkunde, Universitätsklinikum Greifswald, Greifswald 16/17, 17/18, 18/19); A. Stahl (Klinik und Poliklinik für Augenheilkunde, Universitätsklinikum Greifswald, Greifswald 16/17, 17/18, 18/19); A. Huth (Augenklinik, Universitätsklinikum Halle 17/18, 18/19); A. Viestenz (Augenklinik, Universitätsklinikum Halle 17/18, 18/19); P. Bertram (Universitätsklinikum Hamburg-Eppendorf, Klinik f. Augenheilkunde, Hamburg, 16/17, 17/18, 18/19); C. Skevas (Universitätsklinikum Hamburg-Eppendorf, Klinik f. Augenheilkunde, Hamburg, 16/17, 17/18, 18/19); R. Kromer (Universitätsklinikum Hamburg-Eppendorf, Klinikf. Augenheilkunde, Hamburg, 16/17, 17/18, 18/19); M. Casagrande (Universitätsklinikum Hamburg-Eppendorf, Klinik f. Augenheilkunde, Hamburg, 16/17, 17/18, 18/19); C. Grohmann (Universitätsklinikum Hamburg-Eppendorf, Klinikf. Augenheilkunde, Hamburg, 16/17, 17/18, 18/19); J. Mehlan (Universitätsklinikum Hamburg-Eppendorf, Klinik f. Augenheilkunde, Hamburg, 16/17, 17/18, 18/19); P. Schindler (Universitätsklinikum Hamburg-Eppendorf, Klinik f. Augenheilkunde, Hamburg, 16/17, 17/18, 18/19); M. Spitzer 
(Universitätsklinikum Hamburg-Eppendorf, Klinik f. Augenheilkunde, Hamburg, 16/17, 17/18, 18/19); M. Schargus (Augenklinik des Klinikums Asklepios Altona, Hamburg 17/18, 18/19); M. Eddy (Augenklinik des Klinikums Asklepios Altona, Hamburg 17/18, 18/19); S. Schumacher (Augenklinik, Asklepios Klinikum Barmbek, Hamburg 16/17, 17/18, 18/19); M. Keserü (Augenklinik, Asklepios Klinikum Barmbek, Hamburg 16/17, 17/18, 18/19), A. Scheler (Augenklinik, Asklepios Klinikum Barmbek, Hamburg 16/17, 17/18, 18/19); B. Stemplewitz (Augenklinik, Asklepios Klinikum Barmbek, Hamburg 16/17, 17/18, 18/19); U. Schaudig (Augenklinik, Asklepios Klinikum Barmbek, Hamburg 16/17, 17/18, 18/19); W. Abou Moulig (Augenklinik der Medizinischen Hochschule Hannover 17/18, 18/19); C. Framme (Augenklinik der Medizinischen Hochschule Hannover 17/18, 18/19); A. Rosenthal (SLK-Kliniken Heilbronn GmbH, Klinik für Augenheilkunde, Heilbronn 16/17, 17/18); L. Hesse (SLK-Kliniken Heilbronn GmbH, Klinik für Augenheilkunde, Heilbronn 16/17, 17/18); L. Kunz (Augenklinik, Universitätsklinikum des Saarlands, Homburg/Saar 16/17, 17/18, 18/19); C. Spira (Augenklinik, Universitätsklinikum des Saarlands, Homburg/Saar 16/17, 17/18, 18/19); S. Suffo (Augenklinik, Universitätsklinikum des Saarlands, Homburg/Saar 16/17, 17/18, 18/19); B. Seitz (Augenklinik, Universitätsklinikum des Saarlands, Homburg/Saar 16/17, 17/18, 18/19); G. Wietstock (Augenklinik, Medizinische Universität Jena, Jena 18/19); R. Augsten (Augenklinik, Medizinische Universität Jena, Jena 18/19); D. Meller (Augenklinik, Medizinische Universität Jena, Jena 18/19); M. Mayer (Klinik für Augenheilkunde ViDia-Kliniken Karlsruhe 17/18, 18/19); K. Vanselow (Klinik für Augenheilkunde, ViDia-Kliniken Karlsruhe 17/18, 18/19); W. Lieb (Klinik für Augenheilkunde, ViDia-Kliniken Karlsruhe 17/18, 18/19); A. Beutner (Augenklinik, Klinikum Kassel 17/18); R. Effert (Augenklinik, Klinikum Kassel 17/18) C. Ehlken (Universitätsklinikum Schleswig-Holstein, Klinik für Ophthalmologie, Kiel 16/17, 17/18, 18/19); J. Roider (Universitätsklinikum SchleswigHolstein, Klinik für Ophthalmologie, Kiel 16/17, 17/18, 18/19); A. Hueber (Zentrum für Augenheilkunde, Universitätsklinikum Köln 18/19); K. Cursiefen (Zentrum für Augenheilkunde, Universitätsklinikum Köln 18/19); N Schrage (Kliniken der Stadt Köln, Augenklinik Köln-Merheim, Köln 16/17, 17/18, 18/19); M. Kroeger (Helios Klinikum Krefeld, Klinik für Augenheilkunde, Krefeld 16/17, 17/18, 18/19); N. Viehweg (Helios Klinikum Krefeld Klinik für Augenheilkunde, Krefeld 16/17, 17/18, 18/19); P. Meier (Universitätsklinikum Leipzig, Klinik und Poliklinik für Augenheilkunde, Leipzig 16/17, 17/18, 18/19); J.D. Unterlauft (Universitätsklinikum Leipzig, Klinik und Poliklinik für Augenheilkunde, Leipzig 16/17, 17/18, 18/19); P. Wiedemann (Universitätsklinikum Leipzig, Klinik und Poliklinik für Augenheilkunde, Leipzig 16/17, 17/18, 18/19); L. Hattenbach (Klinikum Ludwigshafen gGmbH, Augenklinik, Ludwigshafen 16/17, 18/19); V. Kakkassery (Universitätsklinikum Schleswig-Holstein, Klinik für Augenheilkunde, Lübeck 16/17, 17/8, 18/19); M. Ranjbar (Universitätsklinikum Schleswig-Holstein Klinik für Augenheilkunde, Lübeck 16/17, 17/8, 18/19); A. Mohi (Universitätsklinikum Schleswig-Holstein, Klinik für Augenheilkunde, Lübeck 16/17 17/8, 18/19); S. Grisanti (Universitätsklinikum Schleswig-Holstein, Klinik für Augenheilkunde, Lübeck 16/17, 17/8, 18/19); I. Bastron (Klinikum Lüdenscheid, Augenklinik, Lüdenscheid 16/17, 17/18, 18/19); S. Sarac (Klinikum Lüdenscheid, Augenklinik, Lüdenscheid 16/17, 17/18, 18/19); S. KaskelPaul (Klinikum Lüdenscheid, Augenklinik, Lüdenscheid 16/17, 17/18, 18/19); B. Stoffelns (Augenklinik Universitätsklinikum Mainz 16/17, 17/18, 18/19); A. Schuster (Augenklinik Universitätsklinikum Mainz 16/17, 17/18, 18/19); N. Pfeiffer (Augenklinik Universitätsklinikum Mainz 16/17, 17/18, 18/19); S. Schulze (Augenklinik, Universitätsklinikum Marburg 17/18); A. Praskevas (Augenklinik, Universitätsklinikum Marburg 17/18); W. Sekundo (Augenklinik, Universitätsklinikum Marburg 17/18); M. Weigel (Universitäts-Augenklinik Magdeburg, Magdeburg 16/17); H. Thieme (Universitäts-Augenklinik Magdeburg, Magdeburg 16/17); A. Wolf (Augenklinik und Poliklinik des Klinikums der Universität München, München 16/17, 17/18, 18/19); E. Vounotrypidis (Augenklinik und Poliklinik des Klinikums der Universität München, München 16/17, 17/18, 18/19); S. Priglinger (Augenklinik und Poliklinik des Klinikums der Universität München, München 16/17, 17/18, 18/19); L. Bechstein (Augenklinik des Klinikums der Technischen Universität München 17/18, 18/19); M. Maier (Augenklinik des Klinikums der Technischen Universität München 17/18, 18/19); C. Lohmann (Augenklinik des Klinikums der Technischen Universität München 17/18, 18/19); C. Haritoglu (Augenklinik Herzog Carl Theodor, München 16/17); F. Alten (Universitätsklinikum Münster, Klinik für Augenheilkunde, Münster 16/17, 17/18, 18/19); N. Eter (Universitätsklinikum Münster, Klinik für Augenheilkunde, Münster 16/17, 17/18, 18/19); V. Klishko (Dietrich-Bonhoeffer-Klinikum, Klinik für Augenheilkunde, Neubrandenburg 16/17, 17/18,18/19); U. Hol- land (Dietrich-Bonhoeffer-Klinikum, Klinik für Augenheilkunde, Neubrandenburg 16/17, 17/18,18/19); A. Medra (Dietrich-Bonhoeffer-Klinikum, Klinik für Augenheilkunde, Neubrandenburg 16/17, 17/18,18/19); A. Weber (Dietrich-Bonhoeffer-Klinikum, Klinik für Augenheilkunde, Neubrandenburg 16/17, 17/18,18/19); H. Höh (Dietrich-Bonhoeffer-Klinikum, Klinik für Augenheilkunde, Neubrandenburg 16/17, 17/18,18/19); J. Schmidbauer (Augenklinik, Klinikum Nürnberg Nord 17/18); C. RiveraGomez (Ortenau Klinikum Offenburg-Gengenbach, Augenklinik, Offenburg 16/17, 17/18, 18/19); K. Plantzas (Ortenau Klinikum Offenburg-Gengenbach, Augenklinik, Offenburg 16/17, 17/18, 18/19); M. Weiss (Ortenau Klinikum Offenburg-Gengenbach, Augenklinik, Offenburg 16/17, 17/18, 18/19); K. Hille (Ortenau Klinikum Offenburg-Gengenbach, Augenklinik, Offenburg 16/17, 17/18, 18/19); F. Höhn (Augenklinik, Niels-Stensen-Kliniken, Marienhospital Osnabrück 17/18); S. Aisenbrey (Pius Hospital Oldenburg, Universitätsklinikum Oldenburg, Oldenburg 16/17); M. Schrader (Pius Hospital Oldenburg, Universitätsklinikum Oldenburg, Oldenburg 16/17); A. Napholz (Augenklinik, Helios Klinikum Pforzheim, 18/19); T. Tandogan (Augenklinik, Helios Klinikum Pforzheim, 18/19); C. Walter (Augenklinik Klinikum Ernst-von-Bergmann Potsdam, 16/17, 17/18, 18/19); M. Zühlsdorff-Uthke (Augenklinik Klinikum Ernst-von-Bergmann Potsdam, 16/17, 17/18, 18/19); A. Liekfeld (Augenklinik Klinikum Ernst-von-Bergmann Potsdam, 16/17, 17/18, 18/19); C. Blecha (Universitätsklinikum Regensburg, Klinik und Poliklinik für Augenheilkunde, Regensburg 16/17, 17/18,18/19); T. Barth (Universitätsklinikum Regensburg, Klinik und Poliklinik für Augenheilkunde, Regensburg 16/17, 17/18,18/19); H. Helbig (Universitätsklinikum Regensburg, Klinik und Poliklinik für Augenheilkunde, Regensburg 16/17, 17/18,18/19); A. Juenemann (Universitätsmedizin Rostock, Klinik und Poliklinik für Augenheilkunde, Rostock 16/17, 17/18, 18/19); A. Decker (Augenklinik, Klinikum Saarbrücken 17/18, 18/19); M. Kühn (Augenklinik, Klinikum Saarbrücken 17/18, 18/19); M. Ladewig (Augenklinik, Klinikum Saarbrücken 17/18, 18/19); K. Lenhard (Augenklinik, Katharinenhospital Stuttgart, 18/19); B. Lackner (Augenklinik, Katharinenhospital Stuttgart, 18/19); F. Gekeler (Augenklinik, Katharinenhospital Stuttgart, 18/19); I. Holzschuh (Augenklinik, Klinikum Sulzbach 17/18, 18/19); K.T. Boden (Augenklinik, Klinikum Sulzbach 17/18, 18/19); P. Szurmann (Augenklinik, Klinikum Sulzbach 17/18, 18/19); D. Faul (Krankenhaus der Barmherzigen Brüder Trier, Zentrum für Augenheilkunde, Trier 16/17, 17/18); K. May-Endres (Krankenhaus der Barmherzigen Brüder Trier, Zentrum für Augenheilkunde, Trier 16/17, 17/18); U. Press (Krankenhaus der Barmherzigen Brüder Trier, Zentrum für Augenheilkunde, Trier 16/17, 17/18); J. Luttke (Krankenhaus der Barmherzigen Brüder Trier, Zentrum für Augenheilkunde, Trier 16/17, 17/18); F. Ziemssen (Augenklinik, Universitätsklinikum Tübingen 18/19); U. Bartz-Schmidt (Augenklinik, Universitätsklinikum Tübingen 18/19); J. Cordes (Augenklinik, Universitätsklinikum Ulm, 16/17, 17/18, 18/19); F. Raber (Augenklinik, Universitätsklinikum Ulm, 16/17, 17/18, 18/19); M. Mikielewicz (Augenklinik, Universitätsklinikum Ulm, 16/17, 17/18, 18/19); J. Kammerer (Augenklinik, Universitätsklinikum Ulm, 16/17, 17/18, 18/19); S. Kupferschmid (Augenklinik, Universitätsklinikum Ulm, 16/17, 17/18, 18/19); H. Buchwald (Augenklinik, Universitätsklinikum Ulm, 16/17, 17/18, 18/19); F. Raber (Augenklinik, Universitätsklinikum Ulm, 16/17, 17/18, 18/19); J. Werner (Augenklinik, Universitätsklinikum Ulm, 16/17, 17/18, 18/19); J. Kampmeier (Augenklinik, Universitätsklinikum Ulm, 16/17, 17/18, 18/19); A. Bula (Universitätsklinikum Würzburg, Augenklinik und Poliklinik, Würzburg 16/17, 17/18, 18/19); P. Krauß (Universitätsklinikum Würzburg, Augenklinik und Poliklinik, Würzburg 16/17, 17/18, 18/19); P. Strzalkowski (Universitätsklinikum Würzburg, Augenklinik und Poliklinik, Würzburg 16/17, 17/18, 18/19); J. Hillenkamp (Universitätsklinikum Würzburg, Augenklinik und Poliklinik, Würzburg 16/17, 17/18, 18/19)

\section{Lizenzangabe}

Gabel-Pfisterer, A., Böhringer, D., Agostini, H. et al. Dreijahresergebnisse der deutschlandweiten Umfrage zu Augenverletzungen durch Feuerwerkskörper. Ophthalmologe 2019;116,1138-1151. (DOI: 10.1007/s00347-01900967-9). ${ }^{\circledR} 2019$ Der/die Autor(en) (Reprint, Referenz 24 für de Faber 2019 ergänzt), lizensiert unter CC BY 4.0 (https://creativecommons.org/licenses/by/4.0/deed.de).

\section{Literatur}

Die Literatur ist unter www.karger.com/doi/10.1159/000520208 abrufbar.
180

Kompass Ophthalmol 2021;7:170-180 DOI: $10.1159 / 000520208$ 